\title{
Journal of Nephropathology
}

\section{Effects of atrovastatin on concentrations of 3-hydroxy-3-methyl- glutaryl-coenzyme A- reductase (HMG-CoA-R), proprotein convertase subtilisin/kexin type 9 (PCSK9) and sortilin in patients with type 2 diabetes mellitus and pre-diabetics}

\author{
Ali Nosrati Andevari ${ }^{1,2}$, Soheila Moein ${ }^{1,2^{*}}$, Durdi Qujeq ${ }^{3,4^{*}}$, Zoleika Moazezi ${ }^{5}$, \\ Karimollah Hajian Tilaki ${ }^{\circledR}$
}

\begin{abstract}
${ }^{1}$ Endocrinology and Metabolism Research Center, Hormozgan University of Medical Sciences, Bandar Abbas, Iran ${ }^{2}$ Department of Biochemistry, Faculty of Medicine, Hormozgan University of Medical Sciences, Bandar Abbas, Iran ${ }^{3}$ Cellular and Molecular Biology Research Center, Health Research Institute, Babol University of Medical Sciences, Babol, Iran ${ }^{4}$ Department of Biochemistry, School of Medicine, Babol University of Medical Sciences, Babol, Iran ${ }^{5}$ Department of Internal Medicine, School of Medicine, Babol University of Medical Sciences, Babol, Iran ${ }^{6}$ Department of Statistics and Epidmiology, School of Medicine, Babol University of Medical Sciences, Babol, Iran
\end{abstract}

\begin{tabular}{|c|c|}
\hline ARTICLE INFO & ABSTRACT \\
\hline $\begin{array}{l}\text { Article type: } \\
\text { Original Article }\end{array}$ & $\begin{array}{l}\text { Introduction: Atorvastatin hinders cardiovascular disease by reducing cholesterol levels. Proprotein } \\
\text { convertase subtilisin/kexin type } 9 \text { (PCSK9) enhances the secretion of insulin by binding to LDL- } \\
\text { receptor. Sortilin is committed in the transfer of intracellular proteins through the plasma membrane. }\end{array}$ \\
\hline $\begin{array}{l}\text { Article history: } \\
\text { Received: } 13 \text { April } 2020 \\
\text { Accepted: } 10 \text { June } 2020 \\
\text { Published online: } 2 \text { July } 2020\end{array}$ & $\begin{array}{l}\text { Objectives: The purpose of this research was to determine the effect of atorvastatin consumption } \\
\text { on alterations in the levels of 3-hydroxy-3-methylglutaryl-coenzyme A reductase (HMG-CoA-R), } \\
\text { PCSK9 and sortilin in diabetic patients and pre-diabetics. } \\
\text { Patients and Methods: This study was carried out on } 80 \text { individuals including normal subjects, }\end{array}$ \\
\hline $\begin{array}{l}\text { Keywords: } \\
\text { Diabetics } \\
\text { Pre-diabetics } \\
\text { HMG-COA-R } \\
\text { Sortilin } \\
\text { Atorvastatin } \\
\text { Insulin }\end{array}$ & $\begin{array}{l}\text { diabetic patients and pre-diabetics. The participated individuals were divided as control group (i) } \\
\text { (healthy individuals without diabetes mellitus), diabetic group receiving statin (ii), diabetic group } \\
\text { not receiving statin (iii), pre-diabetic group receiving statin (iv) and pre-diabetic group not receiving } \\
\text { statin (v). Levels of HMG-COA-R, PCSK9 and sortilin were determined by ELISA method. } \\
\text { Results: In diabetics and pre-diabetics taking atorvastatin, the level of HMG-COA-R was not altered } \\
\text { significantly compared to diabetics and pre-diabetics not taking atorvastatin, respectively }(P>0.05) \text {. } \\
\text { The serum PCSK9 level in diabetics and pre-diabetics was significantly higher than the healthy } \\
\text { individuals ( } P=0.001) \text {. Additionally, the serum PCSK9 level in diabetics and pre-diabetics receiving } \\
\text { atorvastatin was significantly higher than diabetics and pre-diabetics not receiving atorvastatin, } \\
\text { respectively ( } P=0.001 \text { ). The serum sortilin level in diabetics and pre-diabetics was significantly } \\
\text { higher than the healthy individuals ( } P=0.001) \text {. In addition, the serum sortilin level in pre-diabetics } \\
\text { receiving atorvastatin was significantly higher than pre-diabetics not receiving atorvastatin } \\
(P=0.001) \text {. } \\
\text { Conclusion: Atorvastatin improved insulin secretion and sensitivity by increasing serum sortilin and } \\
\text { PCSK9 levels. Thereby, it prevented the development of diabetes in diabetics and the progression of } \\
\text { pre-diabetes to diabetes in pre-diabetics. }\end{array}$ \\
\hline
\end{tabular}

Implication for health policy/practice/research/medical education:

In a clinical study on 80 individuals including normal subjects, diabetic patients and pre-diabetics, atorvastatin improved insulin secretion and sensitivity by increasing serum sortilin and PCSK9 levels.

Please cite this paper as: Nosrati Andevari A, Moein S, Qujeq D, Moazezi Z, Hajian Tilaki K. Effects of atrovastatin on concentrations of 3-hydroxy-3-methyl-glutaryl-coenzyme A-reductase (HMG-CoA-R), proprotein convertase subtilisin/kexin type 9 (PCSK9) and sortilin in patients with type 2 diabetes mellitus and pre-diabetics. J Nephropathol. 2021;10(1):e05. DOI: 10.34172/jnp.2021.05.

*Corresponding author: 1. Soheila Moein, Email;soheila_9@yahoo.com, smoein@hums.ac.ir;

2. Durdi Qujeq, Email;dqujeq@gmail.com and D.qujeq@mbabol.ac.ir 


\section{Introduction}

Pre-diabetes is a condition of which blood glucose levels are higher than normal range. However, these levels are less than the threshold for incidence of diabetes (1). Pre-diabetes is a significant risk factor for developing type 2 diabetes (2). Type 2 diabetes occurs because of the incapability of the body to synthesis sufficient insulin or the incapability to use insulin effectively. In patients with type 2 diabetes, one of the complications is the development of cardiovascular disease (CVD) $(3,4)$. Statins prevent CVD by reducing cholesterol levels (5). Statins bind to 3-hydroxy-3-methylglutaryl-coenzyme A (HMG-COA) and retard reversibly the action of 3-hydroxy-3-methylglutaryl-coenzyme A reductase (HMG-CoA-R) (5). Proprotein convertases are recognized as serine proteases that are structurally similar to the subtilisin family of bacterial serine protease $(6,7)$.

In 2003, Seidah et al found a new element of the proprotein convertases family (7). It was discovered that the expression of gene encoding this enzyme had been enhanced during apoptosis of brain cells. Since, it was the ninth element of the proprotein convertases family, it was named as proprotein convertase subtilisin/kexin type 9 (PCSK9), besides convertase neural apoptosis-regulated convertase 1 (NARC-1) (8). PCSK9 is mostly expressed in the liver and a little mount in the small intestine, kidney, brain, and smooth muscle cells. The main function of PCSK9 is decreasing cellular low-density lipoprotein receptor (LDL-R). Hence, PCSK9 is one of the main regulators of low-density lipoprotein cholesterol (LDL-c) level (9).

The accumulation of LDL-c in beta cells of the pancreas disrupts insulin secretion (9). PCSK9 prevents the entry of LDL-c into beta cells and consequence, improves insulin secretion by binding to LDLR in the surface of beta-cells (10). Besides, PCSK9 has a significant role in increasing serum LDL-c level and consequence increasing CVDs, and can prevent type 2 diabetes by improving insulin secretion (10). Sortilin, another factor determined in this study, is the primary member of the vacuolar protein sorting 10 proteins (VPS10Ps) family. It is expressed in some cells like neurons, hepatocytes, skeletal muscle cells, adipocytes and heart (11). Sortilin-1 (SORT1) gene is significantly expressed in hepatocytes and has a significant function in lipoproteins metabolism. Sortilin can increase or decrease very low-density lipoprotein cholesterol (VLDL-c) and LDL-c levels (12). Moreover, sortilin has a significant role in the formation of GLUT4 storage vesicles (GSVs) or insulin-responsive vesicles (IRVs) that transports to the plasma membrane and enhances GLUT4 expression. Thus, sortilin can enhance the sensitivity of insulin (13).

\section{Objectives}

The purpose of this research was to determine the effect of atorvastatin administration on alterations serum levels of 3-Hydroxy-3-methylglutaryl coenzyme A (HMG-CoA) reductase, PCSK9 and sortilin in diabetic patients and pre-diabetics.

\section{Patients and Methods \\ Study patients}

Serum samples were gathered from the patients and individuals from May 2016 to September 2018. They included women and men enrolled for diabetes test. The study was performed on healthy individuals without diabetes mellitus referring to medical diagnostic laboratory (Babol, Iran) and patients with type 2 diabetes mellitus and pre-diabetics referring to endocrinology department. Healthy individuals were selected by an endocrinologist. This study was carried out on 80 individuals and classified into five groups: (i) healthy individuals without diabetes mellitus (control group), (ii) patients with type 2 diabetes mellitus receiving atorvastatin, (iii) patients with type 2 diabetes mellitus not taking atorvastatin, (iv) pre-diabetics receiving atorvastatin, (v) pre-diabetics not receiving atorvastatin. The number of subjects in group (iv) was 12, while, the number of patients and subjects in other groups was 17. Groups (iii) and (v), included diabetic patients and pre-diabetic individuals who did not take atorvastatin. The consort template of this study was shown in Figure 1.

However, in groups (ii) and (iv), diabetic patients and pre-diabetics received atorvastatin $20 \mathrm{mg} / \mathrm{d}$ for three months. Following three months, the samples were obtained. The samples of blood from each subject were taken at a volume of $5 \mathrm{~mL}$ in a serum gathering tube and then the serum was separated for determination. The individuals in the control group were chosen among healthy individuals who were not diabetic. They did not even have specific diseases and diabetes. All patients provide their singed informed consent and accepted to take part in the research.

The inclusion criteria included having type 2 diabetes mellitus (FBS 126 $\geq \mathrm{mg} / \mathrm{dL}$ ), pre-diabetes (FBS 100-125 $\mathrm{mg} / \mathrm{dL}$ ), diabetes for three years without administration of insulin. The exclusion criteria included pregnant or breast feeding women, individuals with active infections such as hepatitis B, hepatitis C, human immunodeficiency virus (HIV) and tuberculosis, history of cancer, heart attack in the last six months or peripheral coronary artery disease, steroid therapy such as the use of prednisone, smoking cigarettes and alcohol intake, women taking contraceptives, indigestion and chronic diarrhea, liver, lung, kidney, heart and brain disorders, anemia and diabetic nephropathy, avoiding to sign the informed consent form. The design of the study is a prospective observational cohort study (without intervention). In the current study, HMG-COA-R, PCSK9 and sortilin were 


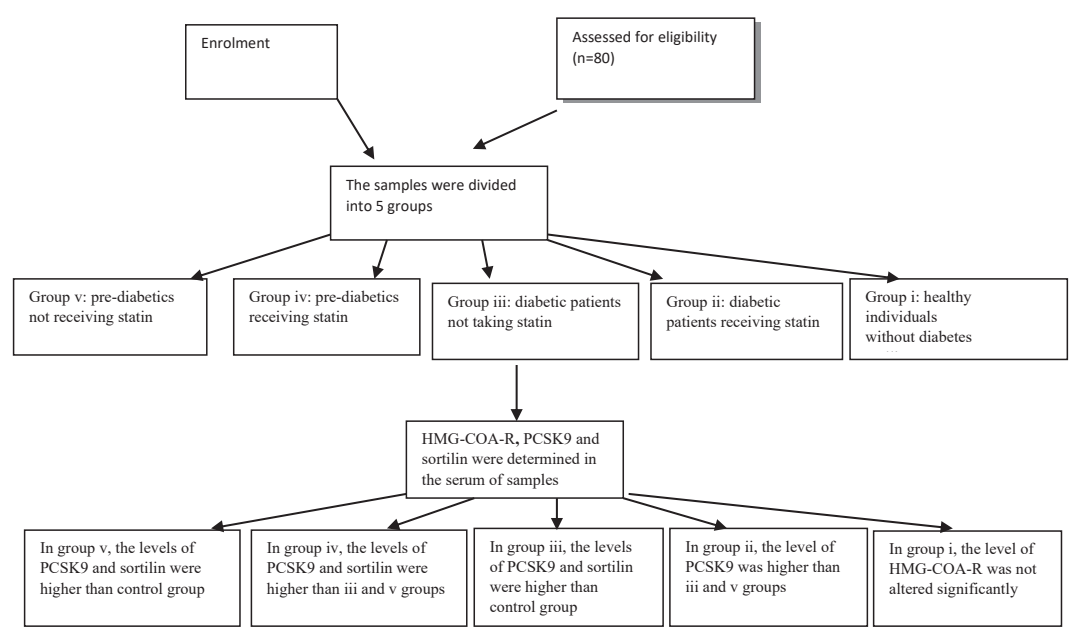

Figure 1. Consort chart of the study included the results of alteration of the studied variables in 5 groups.

measured using ELISA kits at $450 \mathrm{~nm}$.

\section{Ethical issue}

The study was performed in agreement with the principles of Declaration of Helsinki, 1996 version and its later amendments and also Good Clinical Practice standards. The study proposal and written consent procedures were approved by the Ethics Committee at Hormozgan University of Medical Sciences (HUMS.REC.1395.127). This study was the result of M.S of Clinical Biochemistry at this university, conducted by the first author (ANA). This study was carried out in collaboration with Biochemistry Department of Babol University of Medical Sciences.

\section{Statistical analysis}

Data obtained from the study were determined by descriptive statistics (middle, mean, standard deviation) and the correlation between variables and biochemical factors was calculated based on the Pearson's correlation coefficient. The obtained results were analyzed using SPSS version 24. The analysis of variance (ANOVA) test was applied to calculate the mean of data and the box plot was applied to compare the middle of data. Then, the specificity and sensitivity of the variables was calculated using ROC test. $P<0.05$ was statistically considered significant.
Results

In Table 1, the mean and standard deviation of the variables was compared among the five groups based on the ANOVA test. The serum levels of HMG-COA-R in diabetics and pre-diabetics taking statin was not significantly more than diabetics and pre-diabetics no taking statin, $(P>0.05$; Table 1$)$. The levels of PCSK9 in diabetics and pre-diabetics was significantly more than healthy subjects $(P=0.001$; Table 1$)$. Additionally, the serum PCSK9 levels in diabetics and pre-diabetics receiving statin were significantly higher than diabetics and prediabetics not receiving statin, respectively $(P=0.001)$. The serum sortilin levels in diabetics and pre-diabetics were significantly higher than healthy individuals $(P=0.001)$. Furthermore, the serum sortilin level in pre-diabetics receiving statin was significantly higher than pre-diabetics not receiving statin $(P=0.001$; Table 1$)$. The correlation between measured variables in group (i) was shown based on Pearson's test. According to this test, the serum PCSK9 level had positive and significant relationships with the serum sortilin and HMG-COA-R levels $(P=0.001, \mathrm{R}$ $=0.8$ and $P=0.001, \mathrm{R}=0.79$, respectively). Moreover, the serum sortilin level had a positive and significant correlation with serum HMG-COA-R level $(P=0.001$, $\mathrm{R}=0.87$ ).

The correlation between measured variables in group (ii) was shown based on Pearson's test. According to this

Table 1. Comparison of mean and standard deviation of variables measured in 5 groups, according to ANOVA test

\begin{tabular}{|c|c|c|c|c|c|c|}
\hline Variables & $\begin{array}{l}\text { Control } \\
\text { group }\end{array}$ & $\begin{array}{l}\text { Diabetic group } \\
\text { receiving statin }\end{array}$ & $\begin{array}{l}\text { Diabetic group not } \\
\text { receiving statin }\end{array}$ & $\begin{array}{l}\text { Pre-diabetic group } \\
\text { receiving statin }\end{array}$ & $\begin{array}{l}\text { Pre-diabetic group } \\
\text { not receiving statin }\end{array}$ & $P$ value \\
\hline Sortilin (mean $\pm \mathrm{SD}$ ), $\mathrm{ng} / \mathrm{mL}$ & $8 \pm 4$ & $9 \pm 5$ & $9 \pm 4$ & $11 \pm 7$ & $9 \pm 5$ & 0.001 \\
\hline PCSK9 (mean \pm SD), ng/mL & $159 \pm 57$ & $211 \pm 76$ & $189 \pm 72$ & $210 \pm 70$ & $192 \pm 93$ & 0.001 \\
\hline HMG-COA-R (mean \pm SD), ng/mL & $42 \pm 16$ & $41 \pm 16$ & $40 \pm 14$ & $43 \pm 15$ & $41 \pm 11$ & 0.001 \\
\hline
\end{tabular}


Table 2. ROC test between group (i) and group (v)

\begin{tabular}{llllll}
\hline Variable & Sensitivity & Specificity & AUC & Cut off & $P$ value \\
\hline HMG-COA-R & $70 \%$ & $61 \%$ & 0.62 & 35.58 & 0.001 \\
\hline AUC; area under curve. & & & &
\end{tabular}

test, the serum sortilin level had positive and significant correlations with serum PCSK9 and HMG-COA-R levels ( $P=0.001, \mathrm{R}=0.78$ and $P=0.001, \mathrm{R}=0.78$, respectively). Moreover, the serum HMG-COA-R level had a positive and significant correlation with serum PCSK9 levels $(P=0.001, \mathrm{R}=0.89)$.

The correlation between measured variables in group (iii) was shown based on Pearson's test. According to this test, the serum HMG-COA-R level had a positive and significant correlation with the serum PCSK9 level $(P=0.001, \mathrm{R}=0.74)$. Furthermore, there were positive and significant correlations among HMG-COA-R, PCSK9 and sortilin.

The correlation between measured variables in group (iv) was shown based on Pearson's test. According to this test, the serum HMG-COA-R level had positive and significant correlations with the serum sortilin and PCSK9 levels ( $P=0.001, \mathrm{R}=0.92$ and $P=0.001, \mathrm{R}=0.88$, respectively). In addition, the serum sortilin level had a positive and significant correlation with the serum PCSK9 level $(P=0.001, \mathrm{R}=0.87)$. The correlation between measured variables in group (v) was assessed based on Pearson's test. According to this test, serum sortilin level had positive and significant correlations with the serum levels of PCSK9 and HMG-COA-R $(P=0.001, \mathrm{R}=0.81$ and $P=0.001, \mathrm{R}=0.92$, respectively). Additionally, the serum PCSK9 level had a positive and significant correlation with serum HMG-COA-R level $(P=0.001$, $\mathrm{R}=0.82$ ).

In the present study, the middle of data was compared using the box plot. In box plot, control group (i), diabetic group receiving statin (ii), diabetic group not receiving statin (iii), pre-diabetic group receiving statin (iv) and pre-diabetic group not receiving statin (v) were shown. According to Figure 2A, HMG-COA-R level in diabetic group taking statin was more than the control group. The serum PCSK9 level in pre-diabetic group receiving statin was higher than the other groups (Figure 2B). Moreover, the serum level of sortilin in pre-diabetic group taking statin was more than the other groups (Figure 2C).

Figure 2D shows receiver operating characteristic curve (ROC curve) in assessing the specificity and sensitivity of determined factors between group (i) and group (v) for the forecast of the chance of progression from a healthy individual to a pre-diabetic. HMG-COA-R was significantly able to predict passing the healthy phase to the pre-diabetic phase.

ROC curve in assessing the sensitivity and specificity of measured variables between group (i) and group (v) predicted the probability of progression from a healthy
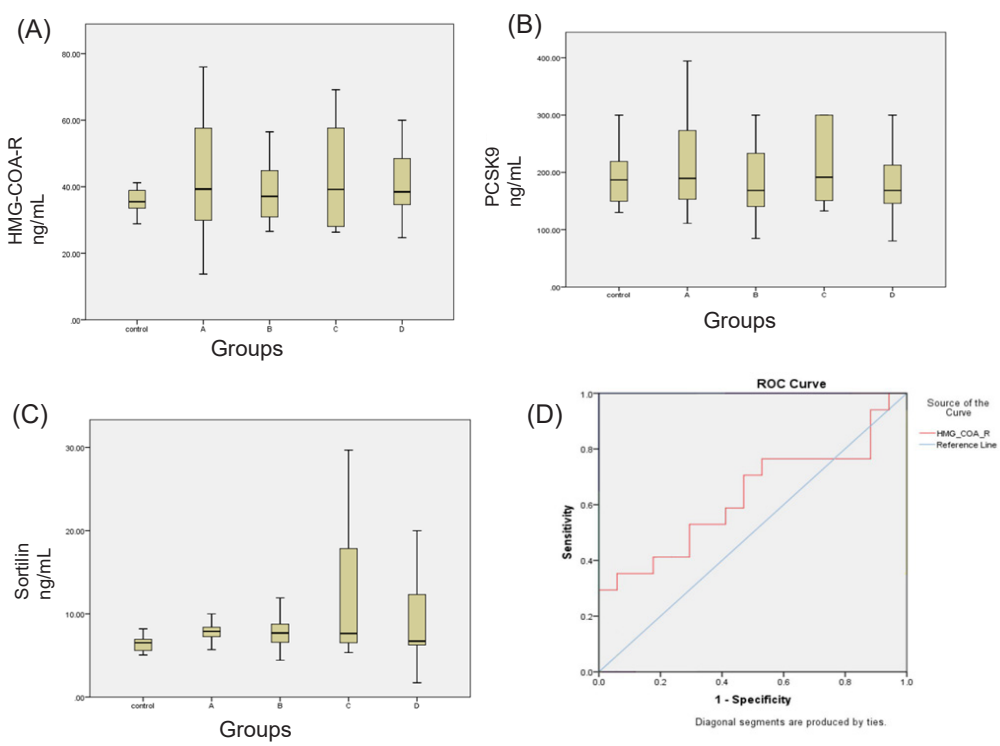

Figure 2. (A) Serum HMG-COA-R level in five groups according to the box plot. (B) Serum PCSK9 level in five groups, according to the box plot. (C) Serum sortilin level in five groups, according to the box plot. (D) Roc curve between group (i) and group (v).

Control group: healthy individuals without diabetes mellitus, A: patients with type 2 diabetes mellitus receiving statin, B: patients with type 2 diabetes mellitus not taking statin, C: pre-diabetics receiving statin, D: pre-diabetics not receiving statin. 
person to a pre-diabetic (Table 2). HMG-COA-R was significantly able to predict the passing of the healthy phase to the pre-diabetic phase with sensitivity of $70 \%$, specificity of $61 \%$ and cut off of $35.58 \mathrm{ng} / \mathrm{dL}$.

\section{Discussion}

The results of the present study demonstrated that the serum PCSK9 level in diabetic and pre-diabetic groups was significantly more than the control group. Given this finding, increased the serum LDL-c and cholesterol concentrations in diabetics and pre-diabetics may be attributed to a rise in the serum level of PCSK9. Additionally, atorvastatin increased the serum PCSK9 level in diabetics and pre-diabetics. In agreement with this result, Mayne et al declared that taking atorvastatin $10 \mathrm{mg} / \mathrm{d}$ for six weeks had significantly enhanced the serum level of PCSK9 (7.4\%) (14). Statins can increase expression of the sterol regulatory element-binding protein 2 (SREBP-2) transcription factor by decreasing the cellular level of cholesterol. SREBP-2 binds to sterol regulatory element- 1 in the PCSK9 gene promoter and as a result, enhances the PCSK9 expression (15). Therefore, the rise of the PCSK9 can limit the effect of statins on serum PCSK9 and LDL-c level, as the natural inhibitor of statins.

It has been shown that the use of PCSK9 inhibitors in combination with statin could enhance the effect of statins in decreasing LDL-c level (16). In another study, it was reported that statins may involve in enhancing insulin secretion and thereby reducing serum level of LDL-c (17).

The results of the ROC test (Table 2) between group (i) and group (v) declared that if the serum level of HMGCOA-R was less than $35.58 \mathrm{ng} / \mathrm{dL}$, it shows pre-diabetic status.

In the present study, the serum level of PCSK9 had a positive and significant correlation with serum levels of HMG-COA-R as shown in Table 1. The reasons for these positive and significant correlations are that SREBP-2 (sterol regulatory element-binding protein 2) can also increase the expression of the HMG-COA-R gene. In addition to the PCSk9 gene, Wu et al declared that the activation of SREBP-2 had been accompanied by enhancement in the HMG-COA-R mRNA expression (18).

In the present study, no relationship between the serum level of sortilin and the serum level of LDL-c was seen. This result may be attributed to the dual role of sortilin in rising or reducing LDL-c level. Furthermore, the serum level of sortilin in diabetic and pre-diabetic groups was substantially more than the control group.

This result showed that the raised lipoproteins in diabetics and pre-diabetics enhanced the expression of sortilin by a mechanism of positive feedback and statin appeared to influence the SORT1 expression. Thereby, the consumption of statin in the pre-diabetic group led to rise in level of sortilin.

The interesting point identified in the present study was the positive and important relationship between serum sortilin, PCSK9 levels and HMG-COA-R. Gustafsen et al showed a positive and significant correlation between serum sortilin level and serum PCSK9 level. They proposed a model in which sortilin had banded to PCSK9 in Golgi apparatus and had facilitated its secretion (19). The correlation between serum sortilin level and serum level of HMG-COA-R has not been considered yet. Our study proved this correlation for the first time as well (Table 1). As mentioned previously, PCSK9 prevents the entry of LDL-c into beta cells and consequence enhances secretion of insulin by binding to LDLR in the surface of beta cells (10).

Sortilin has significant roles in the arrangement of GSVs or IRVs, transfer of these vesicles to the plasma membrane, increases the GLUT4 expression and thereby improves insulin sensitivity (13). Additionally, there was a positive and significant relationship between serum level of sortilin and serum level of PCSK9. Therefore, statin improved the secretion of insulin and sensitivity by increasing the serum sortilin and PCSK9 levels. However, the use of inhibitors of PCSK9 along with atorvastatin therapy can increase atorvastatin effect on reducing LDL-c level while insulin secretion may be impaired.

\section{Conclusion}

Atorvastatin improved insulin secretion and sensitivity by increasing serum sortilin and PCSK9 levels. As a result, it prevented the development of diabetes in diabetics and progression of pre-diabetes to diabetes.

\section{Acknowledgments}

We are grateful to Mr. Mir and Karimian for technical assistance in biochemical assays.

\section{Authors' contribution}

DQ designed the study. AN carried out the experiments. $\mathrm{KH}$ analyzed the data and SM contributed to the writing and revising of the manuscript.

\section{Conflicts of interest}

The authors declare that there are no conflicts of interest regarding the publication of this article.

\section{Ethical considerations}

Ethical issues (including plagiarism, data fabrication, double publication) have been completely observed by the authors. 


\section{Funding/Support}

This project has been partially funded by Hormozgan University of Medical Sciences Bandar Abbas, Iran (\#950180). The other part of this study was financed by Prof Soheila Moein.

\section{References}

1. Twigg SM, Kamp MC, Davis TM, Neylon EK, Flack JR.Prediabetes: a position statement from the Australian Diabetes Society and Australian Diabetes Educators Association. Med J Aust. 2007;186(9):461-465.

2. Norris SL, Zhang X, Avenell A, Gregg E, Bowman B, Schmid $\mathrm{CH}$, et al. Long-term effectiveness of weight-loss interventions in adults with pre-diabetes: a review. Am J Prev Med. 2005;28(1):126-39. doi:10.1016/j.amepre.2004.08.006.

3. Colhoun HM, Betteridge DJ, Durrington PN, Hitman GA, Neil HAW, Livingstone SJ, et al. Primary prevention of cardiovascular disease with atorvastatin in type 2 diabetes in the Collaborative Atorvastatin Diabetes Study (CARDS): multi center randomized placebo-controlled trial. The Lancet. 2004;364(9435):685-696. doi: 10.1016/S01406736(04)16895-5.

4. Harpal S Buttar, DVM, Timao Li, Nivedita Ravi. Prevention of cardiovascular diseases: Role of exercise, dietary interventions, obesity and smoking cessation. Exp Clin Cardiol. 2005;10(4):229-49.

5. Adam O, Laufs U. Rac1-mediated effects of HMG-CoA reductase inhibitors (statins) in cardiovascular disease. Antioxid Redox Signal. 2014;20(8):1238-1250. doi: 10.1089/ars.2013.5526.

6. Naureckiene S, Ma L, Sreekumar K, Purandare U, Lo CF, Huang Y, et al. Functional characterization of Narc 1, a novel proteinase related to proteinase K. Arch Biochem Biophys. 2003;420(1):55-67. doi: 10.1016/j.abb.2003.09.011.

7. Seidah NG, Prat A. The biology and therapeutic targeting of the proprotein convertases. Nat Rev Drug Discov. 2012;11(5):367-383. doi: 10.1038/nrd3699.

8. Rousselet E, Benjannet S, Hamelin J, Canuel M, Seidah NG. The Proprotein Convertase PC7 unique zymogen activation and trafficking pathways. J Biol Chem. 2011;286(4):272838. doi: $10.1038 / \mathrm{nrd} 3699$.

9. Seidah NG, BenjannetS, Wickham L, Marcinkiewicz J, Jasmin SB, Stifani S, et al. The secretory proprotein convertase neural apoptosis-regulated convertase 1 (NARC-1): Liver regeneration and neuronal differentiation. Proc Natl Acad Sci. 2003;100(3):928-33. doi: 10.1073/pnas.0335507100.
10. Zhang DW, Lagace TA, Garuti R, Zhao Z, McDonald M, Horton JD, et al. Binding of PCSK9 to EGF-A repeat of LDL receptor decreases receptor recycling and increases degradation. J Biol Chem. 2007;282:18602-12. doi: 10.1074/jbc.M702027200.

11. He NY, Li Q, Wu CY, Ren Z, Gao Y, Pan LH, et al. Lowering serum lipids via PCSK9-targeting drugs: current advances and future perspectives. Acta Pharmacol Sin. 2017;38(3): 301. doi: 10.1038/aps.2016.134.

12. Wasse FH, Bissonnette S, Saint-Pierre N, Lamantia V, Cyr Y, Chrétien M, et al. The apoB-to-PCSK9 ratio: a new index for metabolic risk in humans. J Clin Lipidol. 2015;9(5): 664675. doi: 10.1016/j.jacl.2015.06.012.

13. Nielsen MS, Madsen P, Christensen EI, Nykjær A, Gliemann J, Kasper D, et al. The sortilin cytoplasmic tail conveys Golgi-endosome transport and binds the VHS domain of the GGA2 sorting protein. EMBO J. 2001;20(9): 21802190. doi: 10.1093/emboj/20.9.2180.

14. Mayne J, Dewpura T, Raymond A, Cousins M, Chaplin A, Lahey KA, et al. Plasma PCSK9 levels are significantly modified by statins and fibrates in humans. Lipids Health Dis. 2008;7:1-9. doi: 10.1186/1476-511X-7-22

15. Zhong LY, Cayabyab FS, Tang CK, Zheng XL, Peng TH, Lv YC. Sortilin: a novel regulator in lipid metabolism and atherogenesis. Clin Chim Acta. 2016;460:11-7. doi: 10.1016/j.cca.2016.06.013.

16. Awan Z, Seidah NG, MacFadyen JG, Benjannet S, Chasman DI, Ridker PM, et al. Rosuvastatin, proprotein convertase subtilisin/kexin type 9 concentrations, and LDL cholesterol response: the Jupiter trial. Clin Chem. 2012;58(1):183-189. doi:10./1373/clinchem.2011.172932.

17. Dubuc G, Chamberland A, Wassef H, Davignon J, Seidah NG, Bernier L, et al. Statins upregulate PCSK9, the gene encoding the proprotein convertase neural apoptosis-regulated convertase-1 implicated in familial hypercholesterolemia. Arterioscler Thromb Vasc Biol. 2004;24(8):1454-9. doi: 10.1161/01.ATV.0000134621.14315.43.

18. Wu N, Sarna LK, Hwang SY, Zhu Q, Wang P, Siow YL, et al. Activation of 3-hydroxy-3-methylglutaryl coenzyme A (HMG-CoA) reductase during high fat diet feeding. Biochim Biophys Acta. 2013;1832(10): 1560-1568. doi: 10.1016/j. bbadis.2013.04.024.

19. Gustafsen C, Kjolby M, Nyegaard M, Mattheisen M, Lundhede J, Buttenschøn H, et al. The hypercholesterolemiarisk gene SORT1 facilitates PCSK9 secretion. Cell Metab, 2014;19(2):310-8. doi: 10.1016/j.cmet.2013.12.006.

Copyright $(2021$ The Author(s); Published by Society of Diabetic Nephropathy Prevention. This is an open-access article distributed under the terms of the Creative Commons Attribution License (http://creativecommons.org/licenses/by/4.0), which permits unrestricted use, distribution, and reproduction in any medium, provided the original work is properly cited. 